\title{
Quantitative analysis of betaine in Lycii Fructus by HILIC-ELSD
}

\author{
Bing Tian Zhao $\cdot$ Su Yang Jeong $\cdot$ Kyoung Hwangbo $\cdot$ Dong Cheul Moon \\ Eun-Kyoung Seo $\cdot$ Dongho Lee $\cdot$ Je-Hyun Lee $\cdot$ Byung-Sun Min $\cdot$ \\ Eun-Sook Ma $\cdot$ Jong Keun Son $\cdot$ Mi Hee Woo
}

Received: 23 January 2013/Accepted: 1 May 2013/Published online: 11 May 2013

(C) The Author(s) 2013. This article is published with open access at Springerlink.com

\begin{abstract}
A rapid and simple high-performance liquid chromatography (HPLC) method with evaporative light scattering detection (ELSD) was developed for the determination of betaine from Lycii Fructus. Betaine was separated with an Atlantis hydrophilic interaction liquid chromatography silica column $(4.6 \times 150 \mathrm{~mm}, 5 \mu \mathrm{m}$, $100 \AA$ ) by isocratic elution using $30 \mathrm{mM}$ ammonium acetate buffer and acetonitrile $(20: 80, \mathrm{v} / \mathrm{v} \%)$ as the mobile phase. The flow rate was $1.0 \mathrm{~mL} / \mathrm{min}$, and the temperature for the spray chamber and drift tube was set at 30 and $50{ }^{\circ} \mathrm{C}$, respectively. The method was fully validated with respect to linearity, precision, accuracy, stability and
\end{abstract}

B. T. Zhao $\cdot$ S. Y. Jeong $\cdot$ B.-S. Min · E.-S. Ma

M. H. Woo ( $\square)$

College of Pharmacy, Catholic University of Daegu, Gyeongsan

712-702, South Korea

e-mail: woomh@cu.ac.kr

K. Hwangbo · J. K. Son $(\varangle)$

College of Pharmacy, Yeungnam University, Gyeongsan 712-

749, South Korea

e-mail: jkson@yu.ac.kr

D. C. Moon

College of Pharmacy, Chungbuk National University, Cheongju

361-763, South Korea

E.-K. Seo

The Center for Cell Signaling \& Drug Discovery Research, College of Pharmacy, Ewha Womans University, Seoul 120-750, South Korea

D. Lee

School of Life Sciences and Biotechnology, Korea University, Seoul 136-701, South Korea

J.-H. Lee

College of Oriental Medicine, Dongguk University, Gyeongju

780-714, South Korea robustness. The HPLC/ELSD method was applied successfully to the quantification of betaine in the extract of Lycii Fructus. The HPLC analytical method for pattern recognition analysis was validated by repeated analysis of twenty-six L. barbarum L. from China (BC01-BC26), $3 \mathrm{~L}$. barbarum L. (BJ27-BJ29) from Japan, 12 L. chinense Miller from China (CC30-CC41) and 51 L. chinense Miller samples (CK42-CK92) from Korea. The results indicate that the established HPLC/ELSD method is suitable for quality evaluation of Lycii Fructus.

Keywords Lycii Fructus - Betaine - HPLC - ELSD . Pattern recognition - Quality control

\section{Introduction}

Lycii Fructus, the dried ripe fruits of Barbary wolfberry, belongs to the Solanaceae family (Adams et al. 2006). It has a long history of applications as a valuable tonic medicine and health food supplement for improving vision and maintaining good health. Lycii Fructus is reputed to have the properties of nourishing the blood, enriching the yin, tonifying the kidney and liver, and moistening the lungs (Peng et al. 2005). Traditionally, Lycii Fructus was used as sources for herbal drugs, medicinal vegetables, and functional teas in China, Europe, North America, and Southeast Asia (Duan et al. 2010). Recently, studies have found that Lycii Fructus possesses various characteristics including antioxidant, antiaging, antitumor, cytoprotective, neuroprotective, antiglaucoma and immunomodulatory activities, and promotes endurance and improves control of glucose and other diabetic symptoms (Zhang et al. 2010; Li et al. 2007, 2011; Tang et al. 2011).

Betaine, which is a natural amino-acid and one of the major functional components in Lycii Fructus, has been 
proven to be involved in the process of anti-atherosclerosis, anti-osteoporosis and against chemical liver injury etc. (Michels 2011). In addition, the Korean Pharmacopoeia (K.P. 2007) stipulates that Lycii Fructus contains no $<0.5 \%$ of betaine from L. barbarum L. or L. chinense Miller. However, the content regulation is different between K.P. and Chinese Pharmacopoeia (C.P. 2010); In C.P., it is prescript to contain no less than $0.3 \%$ of betaine and $1.8 \%$ of L. barbarum-polysaccharides from only one species of $L$. barbarum L. In other words, the content regulation of betaine is significantly different between C.P. and K.P. The K.P. content regulation of betaine is more than 1.5 times higher than that of C.P. Additionally, the present HPLC-UV method for the determination of betaine by K.P. (IX) needs to be revised due to the complex sample processing and the unreasonable UV detector at $210 \mathrm{~nm}$. The purpose of this study was to establish a stable HPLC method which could quantitatively analyze major compounds from Lycii Fructus, and to suggest the analytical method determined from this work as an official analytical method in K.P.

Inbaraj et al. (2008) used HPLC-DAD-APCI-MS to determine 11 free carotenoids and 7 carotenoid esters in the fruits of L. barbarum. Furthermore, several analytical approaches, such as liquid chromatography (LC), gas chromatography-mass spectrometry (GC-MS) and HPLC method, had been developed to determine the content of betaine in Lycii Fructus. Shin et al. (1999) developed a rapid and sensitive LC-electrospray ionization-mass spectrometry (LC-EI-MS) for the determination of betaine in $L$. chinense fruits, in which complicated ion exchange processes are required to remove most of the amino acids that could interfere with the quantification of betaine. $\mathrm{Xu}$ et al. (2006) developed a HPLC/UV method using derivatization reaction to determine betaine in the granules of Fufang Guilu. Huang et al. (2010) developed a HPLC/ELSD method using a Luna SCX (Strong Cation Exchange) $100 \AA$ column for the determination of betaine in Lycii Fructus, which was unreasonable due to the bad resolution having very short retention time (about $5 \mathrm{~min}$ ). Lee et al. (2011) developed an ion-pair reversed-phased LC (IPRPLC) with long perfluoropentanoic acid as the volatile ion-pairing reagent on an octadecyl silica (ODS) column and an ELSD. However, betaine peak was not well separated from other peaks, because the ion-pairing reagent of alkyl chain could be irreversibly adsorbed to the ODS C18 stationary phase. Shin et al. (2012) used an HILIC column with an ELSD detector to determine betaine in Lycii Fructus using complex gradient mobile elution. In addition, they classified Lycii Fructus into three different groups according to their geographical origins using only 14 Lycii Fructus samples, which was different from our results from 92 Lycii Fructus samples. On the other hand, to determine betaine in Lycii Fructus, we developed a fast, accurate and simple HPLC/ELSD method using HILIC column with an isocratic elution.

The present paper describes a suitable method for quantitation and pattern recognition analyses for quality evaluation of Lycii Fructus. All of the published HPLC studies had been focused only on the fruits of $L$. barbarum L. There are no reports on the fruits of L. chinense Miller, which also belongs to Lycii Fructus in K.P. Therefore, we used the fruit samples of both $L$. barbarum L. and $L$. chinense Miller in this study.

\section{Materials and methods}

Chemicals and materials

The betaine standard was kindly provided by Professor Eun-Kyoung Seo (Ewha Womans University, Seoul, Korea), which was separated from $L$. chinense Miller (CK42). The internal standard (I.S.) of $\beta$-alanine was purchased from Junsei chemical. The compound structures are shown in Fig. 1. The purities of these compounds were determined to be greater than $98 \%$ by normalization of the peak areas detected by HPLC analyses. Acetonitrile was purchased from Merck K GaA (Darmstadt, Germany). All other chemicals used were of analytic grade unless otherwise noted. Distilled water was prepared using the Milli-Q purification system (Millipore, Bedford, MA, USA). This study adopted the fruit samples of 26 L. barbarum L. from China (BC01-BC26), 3 L. barbarum L. (BJ27-BJ29) from Japan, 12 L. chinense Miller from China (CC30-CC41), 51 L. chinense Miller samples (CK42-CK92) were cultured from Korea. These samples cultured in different regions were provided by the National Center for Standardization of Herbal Medicine.

\section{Sample preparation}

A standard stock solution was prepared by adding $2.0 \mathrm{mg}$ of betaine in $1.0 \mathrm{~mL}$ of $\mathrm{H}_{2} \mathrm{O}$ containing I.S. solution (1,000 ppm of $\beta$-alanine) and then diluted with I.S. solution to obtain the appropriate concentration of working solution.

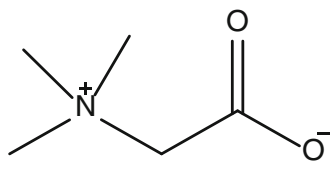

betaine<smiles>NCCC(=O)O</smiles>

$\beta$-alanine (I.S.)
Fig. 1 Structures of betaine and an internal standard 

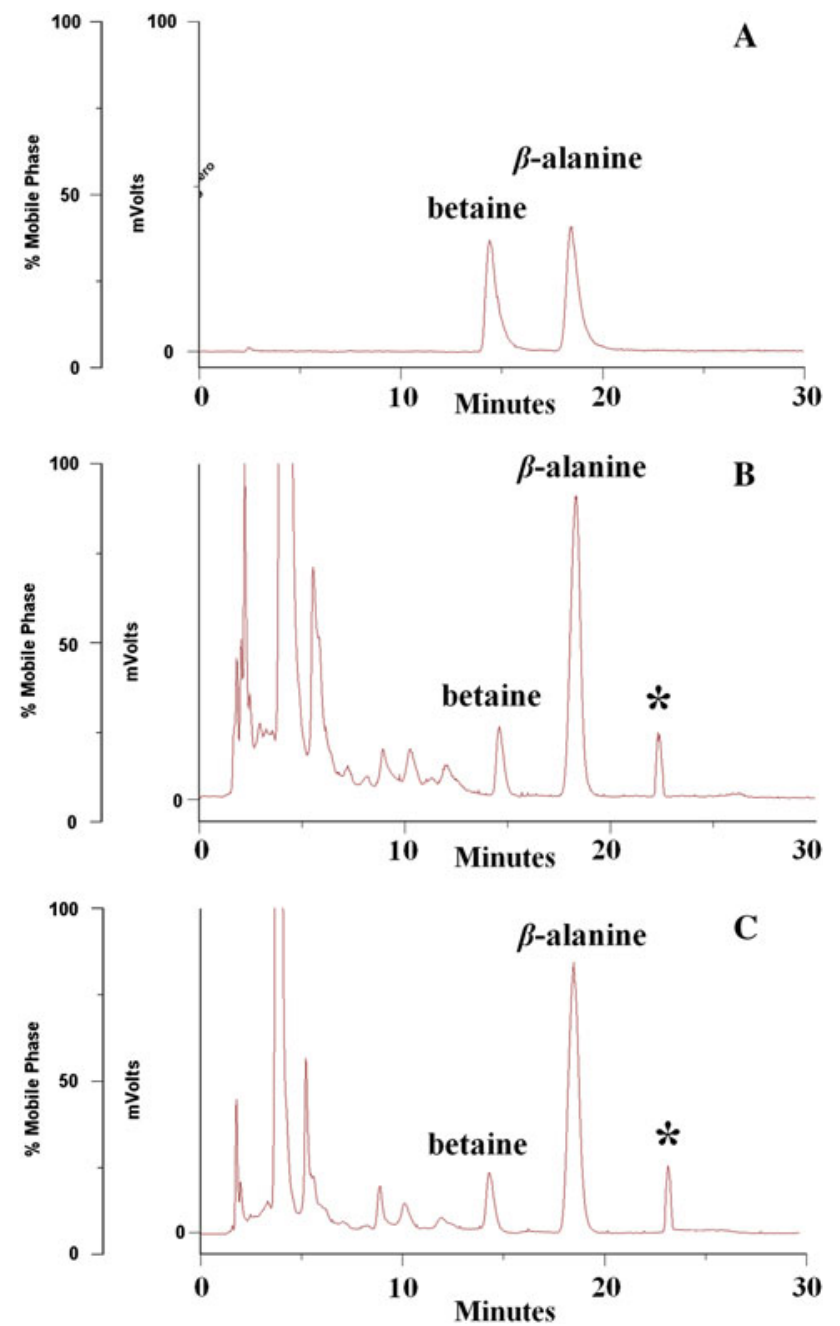

Fig. 2 HPLC chromatograms of standard mixture (a), the sample of $L$. barbarum L (BC01; b) and $L$. chinense Miller (CK50; c). *Unknown peak for pattern recognition analysis

A powder sample of Lycii Fructus (1.0 g) was mixed with $50 \mathrm{~mL}$ of $\mathrm{H}_{2} \mathrm{O}$ containing $1,000 \mathrm{ppm}$ of I.S. in a vial. The mixture was then sonicated for $60 \mathrm{~min}$. The solution was weighed again, and the loss in weight was made up with $\mathrm{H}_{2} \mathrm{O}$. The solution was filtered through a $0.21-\mu \mathrm{m}$ membrane filter (Whatman) and the filtrate was used as the test solution. $10 \mu \mathrm{L}$ of the test solution was then injected into the HPLC system.

\section{HPLC/UV conditions}

The HPLC equipment was a Gilson HPLC system (Unipoint 2.0) with a Gilson 321 pump, a Gilson Prep TM II
ELSD detector and Gilson 321 XL auto-sampler (Gilson Inc.). Two different columns were used and compared: Atlantis silica HILIC $100 \AA\left(150 \times 4.6 \mathrm{~mm}^{2}, 5 \mu \mathrm{m}\right)$ and Phenomenex Luna HILIC $200 \AA$ (150 $\left.\times 4.6 \mathrm{~mm}^{2}, 5 \mu \mathrm{m}\right)$. The columns were conjugated by the guard column $\left(4.0 \times 3.0 \mathrm{~mm}^{2}\right.$, Security Guard Cartridges $)$. The mobile phase was used as an isocratic elution of acetonitrile and $30 \mathrm{mM}$ of ammonium acetate buffer $(80 / 20, \mathrm{v} / \mathrm{v})$ in 0-30 min. The mobile phase was filtered under vacuum through a $0.21-\mu \mathrm{m}$ membrane filter and was degassed prior to use. The analysis was carried out at a flow rate of $1.0 \mathrm{~mL} / \mathrm{min}$ with ELSD detection. The ELSD parameters of spray chamber, drift tube temperature and gas pressure were optimized at $30,50{ }^{\circ} \mathrm{C}$ and $60 \mathrm{psi}$, respectively.

Analytical method validation

A standard stock solution was prepared and diluted to an appropriate concentration for the construction of calibration. The calibration curve was composed of six concentration levels ranging from 5.0 to $800.0 \mu \mathrm{g} / \mathrm{mL}(5.0,50.0$, $100.0,200.0,400.0$ and $800.0 \mu \mathrm{g} / \mathrm{mL})$. The calibration curve was constructed by plotting the logarithm of the peak area ratio (betaine/I.S.) with the logarithm of six different concentration values. The lowest concentration of working solution was diluted with appropriate concentrations, and the limit of detection (LOD) and quantification (LOQ) under the chromatographic conditions were separately determined at signal-to-noise ratios $(\mathrm{S} / \mathrm{N})$ of about 3 and 10 , respectively.

Recovery was determined by spiking three concentration levels for the standard of betaine, which was mixed with a Lycii Fructus (CK42) sample for subsequent extraction and filtration. The HPLC/ELSD analytical experiments were performed in triplicate for each control level. The data from the standard solution and the extracted sample were compared. Precision and accuracy were determined by multiple analyses $(n=5)$ of quality control samples prepared at low, medium and high concentrations spanning the calibration range.

The robustness of the method was studied by introducing changes in column (i.e. Atlantis, Phenomenex) and separation temperature (i.e. $25,30,35$ and $40^{\circ} \mathrm{C}$ ) and using three different flow rates (i.e. $0.8,1.0$ and $1.2 \mathrm{~mL} /$ $\min )$.

Stability was tested with a mixed standard solution stored in the dark at $4{ }^{\circ} \mathrm{C}$ and at room temperature $\left(25^{\circ} \mathrm{C}\right)$.

Table 1 Linearity, linear ranges, LOD and LOQ

\begin{tabular}{llllll}
\hline Analytes & Regression equation & Linearity range $(\mu \mathrm{g} / \mathrm{mL})$ & Correlation coefficient $\left(r^{2}\right)$ & $\mathrm{LOD}(\mu \mathrm{g} / \mathrm{mL})$ & $\mathrm{LOQ}(\mu \mathrm{g} / \mathrm{mL})$ \\
\hline Betaine & $1.424115 \mathrm{x}+0.000059$ & $5.0-800.0$ & 0.9997 & 2.31 & 7.11 \\
\hline
\end{tabular}


Table 2 Precision and accuracy of analytical results

\begin{tabular}{|c|c|c|c|c|c|c|c|c|c|}
\hline \multirow[t]{2}{*}{ Analyte } & \multirow{2}{*}{$\begin{array}{l}\text { Fortified } \\
\text { conc. } \\
(\mu \mathrm{g} / \mathrm{mL})\end{array}$} & \multirow{2}{*}{$\begin{array}{l}\text { Sample } \\
\text { conc. } \\
(\mu \mathrm{g} / \mathrm{mL})\end{array}$} & \multicolumn{3}{|l|}{ Intra-day $(n=5)$} & \multirow{2}{*}{$\begin{array}{l}\text { Sample conc. } \\
(\mu \mathrm{g} / \mathrm{mL})\end{array}$} & \multicolumn{3}{|l|}{ Inter-day $(n=5)$} \\
\hline & & & $\begin{array}{l}\text { Observed } \\
(\mu \mathrm{g} / \mathrm{mL})\end{array}$ & $\begin{array}{l}\text { Accuracy } \\
(\%)\end{array}$ & $\begin{array}{l}\text { Precision } \\
(\%)\end{array}$ & & $\begin{array}{l}\text { Observed } \\
(\mu \mathrm{g} / \mathrm{mL})\end{array}$ & $\begin{array}{l}\text { Accuracy } \\
(\%)\end{array}$ & $\begin{array}{l}\text { Precision } \\
(\%)\end{array}$ \\
\hline \multirow[t]{3}{*}{ Betaine } & 10 & 209.72 & $219.94 \pm 1.82^{\mathrm{a}}$ & $102.21 \pm 0.84$ & 0.82 & 200.19 & $210.88 \pm 0.73$ & $106.89 \pm 0.36$ & 0.34 \\
\hline & 100 & 209.72 & $311.55 \pm 1.78$ & $101.83 \pm 0.58$ & 0.57 & 200.19 & $300.55 \pm 1.78$ & $100.36 \pm 0.47$ & 0.47 \\
\hline & 1000 & 209.72 & $1284.94 \pm 1.58$ & $107.52 \pm 1.05$ & 0.98 & 200.19 & $1193.35 \pm 1.75$ & $99.32 \pm 0.31$ & 0.31 \\
\hline
\end{tabular}

${ }^{\mathrm{a}}$ Mean $\pm \mathrm{SD}$ (standard deviation; $\mathrm{n}=5$ )

Table 3 Robustness of column, temperature and flow rate on the theoretical plate $(N)$, capacity factor $\left(k^{\prime}\right)$, separation factor $(\alpha)$ and resolution $(R s)$

\begin{tabular}{lllrrr}
\hline & Conditions & \multicolumn{1}{l}{$N$} & \multicolumn{1}{l}{$\alpha$} \\
\hline Column & Atlantis silica HILIC & $5.18 \pm 0.07^{\mathrm{a}}$ & $1544.26 \pm 44.19$ & $1.28 \pm 0.00$ & $6.16 \pm 0.18$ \\
& Phenomenex Luna HILIC & $2.34 \pm 0.03$ & $836.14 \pm 41.57$ & $1.22 \pm 0.01$ & $5.39 \pm 0.27$ \\
Temperature $\left({ }^{\circ} \mathrm{C}\right)$ & 25 & $5.18 \pm 0.07$ & $1544.26 \pm 44.19$ & $1.28 \pm 0.00$ & $6.16 \pm 0.18$ \\
& 30 & $5.39 \pm 0.04$ & $1523.65 \pm 40.03$ & $1.29 \pm 0.00$ & $6.65 \pm 0.19$ \\
& 35 & $5.39 \pm 0.07$ & $1588.59 \pm 40.73$ & $1.28 \pm 0.00$ & $6.04 \pm 0.04$ \\
Flow rate $(\mathrm{mL} / \mathrm{min})$ & 40 & $5.39 \pm 0.11$ & $1590.00 \pm 12.74$ & $1.28 \pm 0.01$ & $6.03 \pm 0.07$ \\
& 0.8 & $5.84 \pm 0.03$ & $1518.17 \pm 20.69$ & $1.23 \pm 0.00$ & $6.73 \pm 0.02$ \\
& 1.0 & $5.18 \pm 0.08$ & $1544.26 \pm 44.20$ & $1.28 \pm 0.00$ & $6.16 \pm 0.19$ \\
& 1.2 & $5.66 \pm 0.13$ & $1573.82 \pm 10.34$ & $1.26 \pm 0.01$ & $6.99 \pm 0.08$ \\
\hline
\end{tabular}

${ }^{\mathrm{a}}$ Mean $\pm \mathrm{SD}$ (standard deviation; $n=3$ )

Two samples were analyzed in triplicate at $0,0.5,1,2,5$, 10, 15 and 30 days. The compounds were stable over the tested period (data not shown).

Pattern recognition analysis

To evaluate the phytochemical equivalency among the 92 samples (26 L. barbarum L. from China (BC01-BC26), 3 L. barbarum L. (BJ27-BJ29) from Japan, 12 L. chinense Miller from China (CC30-CC41), 51 L. chinense Miller samples (CK42-CK92) from Korea), pattern recognition analysis was conducted. In this study, we used the peak areas of betaine and one unknown peak to analyze Hclust of pattern recognition analysis using software IBM SPSS Statistics Version 19.

\section{Results and discussion}

Optimization of chromatographic conditions

HPLC conditions were selected according to the requirement for obtaining chromatograms with better resolution of adjacent peaks within a short retention time. For the optimization of chromatographic conditions, we investigated ODS C18 and HILIC columns. Reversedphase of ODS C18 was inappropriate since the betaine peak had a very short retention time due to its physicochemical properties of high polarity. On the detector selection, an ELSD detector was used in this study because its LOD and LOQ levels were about 150 times higher than those of UV detection at the same concentration of betaine (data not shown). The mobile phase composition on the separation was examined for the HILIC column. A mobile phase of water-methanol was inadequate for good resolution of betaine. $30 \mathrm{mM}$ of ammonium acetate buffer and acetonitrile modifier significantly improved the separation for the HILIC column. We also tested adding buffer (ammonium formate and ammonium acetate) at different $\mathrm{pH}$ values $(3.0,5.0,7.0)$ in the mobile phase. The addition of $30 \mathrm{mM}$ ammonium acetate buffer at $\mathrm{pH} 3.0$ to the mobile phase resulted in good resolution of betaine, as well as peak symmetry. Furthermore, we set the ELSD parameters for the spray chamber, drift tube temperature and gas pressure, with the purpose of producing a betaine peak of a good steady and highest pattern. Ultimately, the optimal mobile phase was a mixture of acetonitrile and $30 \mathrm{mM}$ of ammonium acetate buffer $(80 / 20, \mathrm{v} / \mathrm{v})$ in the isocratic elution mode. The typical chromatograms of the sample and standard mixture are shown in Fig. 2; the target compound including an internal standard was completely separated within 30 min. $\beta$-Alanine was selected as an internal standard (Fig. 1). 
Table 4 Content (wt $\%)$ of betaine in Lycii Fructus

\begin{tabular}{|c|c|c|c|}
\hline & \multicolumn{3}{|l|}{ Mean \pm SD $(w t \%)$} \\
\hline & Korean products $(n=51)$ & Chinese products $(n=38)$ & Japanese products $(n=3)$ \\
\hline L. chinense Miller $(n=63)$ & $\begin{array}{l}0.60 \pm 0.40^{\mathrm{a}} \\
\quad(\mathrm{CK} 42-\mathrm{CK} 92 ; n=51)\end{array}$ & $\begin{array}{l}0.74 \pm 0.34 \\
\quad(\mathrm{CC} 30-\mathrm{CC} 41 ; n=12)\end{array}$ & - \\
\hline L. barbarum L. $(n=29)$ & - & $\begin{array}{l}0.58 \pm 0.23 \\
\quad(\mathrm{BC} 01-\mathrm{BC} 26 ; n=26)\end{array}$ & $\begin{array}{l}0.82 \pm 0.18 \\
\quad(\mathrm{BJ} 27-\mathrm{BJ} 29 ; n=3)\end{array}$ \\
\hline
\end{tabular}

${ }^{a}$ Each value represents the mean $\pm \mathrm{SD}(n=3)$
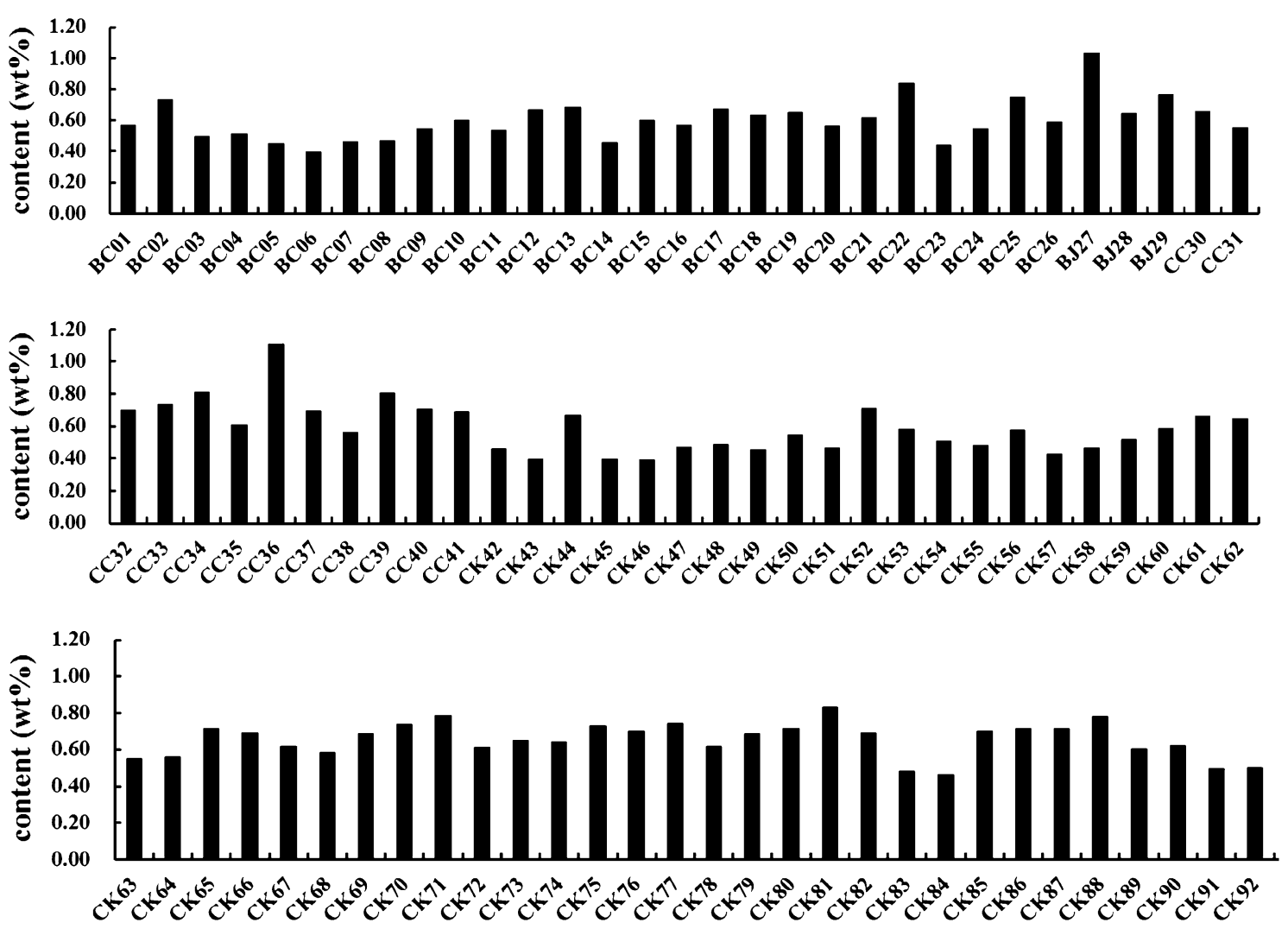

Fig. 3 Graphic presentation of betaine contents (wt $\%, n=3$ ). BC: L. barbarum L. (Chinese product); BJ: L. barbarum L. (Japanese product); CC: L. chinense Miller (Chinese product); CK: L. chinense Miller (Korean product)

Optimization of sample preparation conditions

Five extracting solvents, $70 \%$ ethanol, $50 \%$ ethanol, $70 \%$ methanol, $50 \%$ methanol, and $100 \% \mathrm{H}_{2} \mathrm{O}$, were compared in sample assays after extraction by sonication for $1 \mathrm{~h}$ at room temperature. When the sample was extracted with $100 \%$ $\mathrm{H}_{2} \mathrm{O}$, the sample assay was higher than the other solvent samples. Therefore, we employed $100 \% \mathrm{H}_{2} \mathrm{O}$ as the extracting solvent throughout this work. Two extraction methods, ultra-sonication and reflux using $100 \% \mathrm{H}_{2} \mathrm{O}$ extraction solvent, were compared in sample assays. Extraction by sonication was higher than extraction by reflux in sample assays. To determine the time needed for completion of extraction, samples were extracted for five different periods of time $(30,45,60,90$ and $120 \mathrm{~min})$. Thus, $100 \%$ of $\mathrm{H}_{2} \mathrm{O}$ was employed in the sonication extraction method at room temperature. When the extraction time was set to $60 \mathrm{~min}$, the sample assay results were similar to those with $90 \mathrm{~min}$. Therefore, when the extraction time was $60 \mathrm{~min}$, all the compounds were sufficiently extracted (data not shown).

Validation of the method

Linearity, calibration range, limits of detection and quantification

The calibration curve showed good linear regression $\left(r^{2}\right)>0.999$ within the test ranges, as shown in Table 1. 
Fig. 4 HClust of 92 authentic specimens of Lycii Fructus. Group A (empty circle): L. chinense Miller (CC30-CC41 and CK42-CK92), Group B (filled circle): $L$. barbarum L. (BC01-BC26 and BJ27-BJ29)

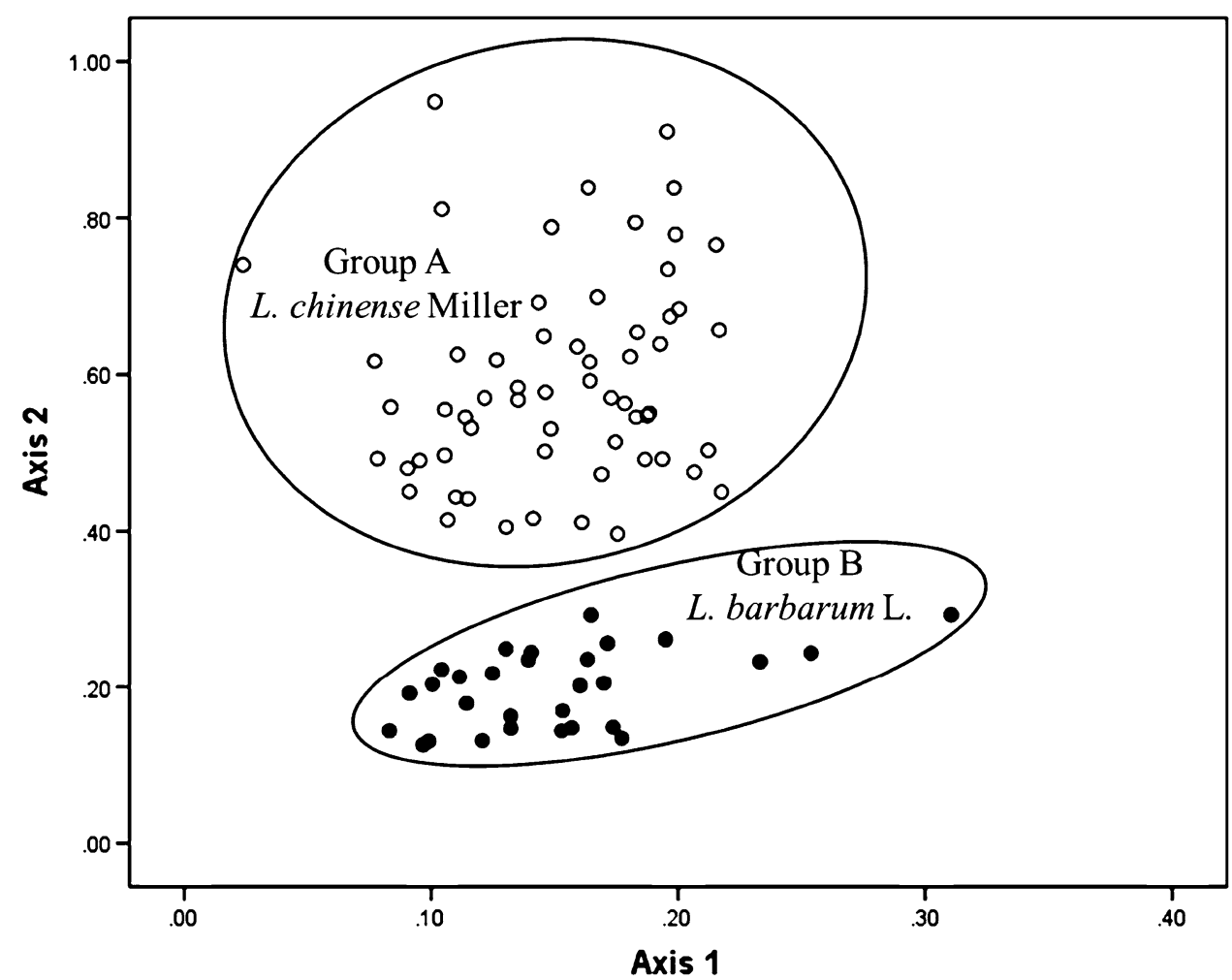

The stock solution containing the reference compound was diluted to give a series of appropriate concentrations with $\mathrm{H}_{2} \mathrm{O}$ and the aliquots of the diluted solutions were injected. $\operatorname{LOD}(\mathrm{S} / \mathrm{N}=3)$ and $\operatorname{LOQ}(\mathrm{S} / \mathrm{N}=10)$ for betaine were found at 2.13 and $7.11 \mu \mathrm{g} / \mathrm{mL}$, respectively. Hence, this verified HPLC-ELSD method was precise, accurate and sensitive enough for quantitative evaluation of major betaine in Lycii Fructus.

\section{Precision and accuracy}

The extraction recovery test was performed by extracting a known amount of betaine from Lycii Fructus powder samples. Known amounts of each standard compound at three levels were mixed with the sample powder and then extracted with $100 \% \mathrm{H}_{2} \mathrm{O}$, as described in the experimental section. The average recovery was represented by the formula: $\mathrm{R}(\%)=[$ (amount from the sample spiked standard - amount from the sample)/amount from the spiked standard] $\times 100$. Intra-assay precision and accuracy were determined from the variability of multiple analyses $(n=5)$ of quality control samples analyzed within the same analytical run. The quality control samples had intraassay precision below $0.98 \%$ and accuracy between $101.83 \%$ and $107.52 \%$. Inter-assay precision and accuracy were evaluated from the variability of multiple analyses $(n=5)$ of quality control samples analyzed for three consecutive days. The quality control samples had an inter- assay precision of $<0.47 \%$ and accuracy between 99.32 and $106.89 \%$. Thus, the developed method can be said to be highly reproducible. The precision and accuracy data are presented in Table 2.

\section{Robustness}

The robustness was determined in order to evaluate the reliability of the established HPLC method. All the parameters were maintained so there would not be any interference with other peaks from Lycii Fructus. The experimental conditions, such as column temperature, column species and flow rates, were purposely altered. The theoretical plate $(\boldsymbol{N})$, capacity factor $\left(k^{\prime}\right)$, separation factor $(\alpha)$ and resolution $(R s)$ were evaluated. In the term of the robustness study for the HPLC assay, four analytical factors did not differ greatly, when the temperature and flow rate conditions were changed. However, four analytical factors of the Atilantis silica HILIC column were significantly superior to the Phenomenex Luna HILIC column during the column performance test (Table 3), since the particle size (100 $\AA$ ) of the Atlantis silica HILIC column was smaller than that $(200 \AA)$ of the Phenomenex Luna HILIC column. These results collectively show that the Atlantis silica HILIC column (100 ̊) was considered to be more suitable for separation of small molecular weight of betaine. Hence, an Atlantis silica HILIC column (100 ̊) was used in the present study. 
Sample analysis

The developed HPLC method was applied to the analysis of 92 Lycii Fructus samples collected from different locations in Korea, China and Japan. In the L. chinense Miller samples, the content $(0.74 \%)$ of betaine from Chinese products was recorded to be higher than that $(0.67 \%)$ of Korean products. On the other hand, in L. barbarum L. samples the content $(0.82 \%)$ of betaine from Japanese products was found to be higher than that $(0.58 \%)$ of Chinese products. In Chinese products, the content $(0.74 \%)$ of betaine in the L. chinense Miller sample was found to be higher than that $(0.58 \%)$ of $L$. barbarum L. The sample analysis results are summarized in Table 4 and Fig. 3.

This quantitative analysis results of betaine for 92 Lycii Fructus samples will be reflected on the content regulation of betaine for Lycii Fructus in the next K.P. revision.

\section{Pattern recognition analysis}

To evaluate the phytochemical equivalency among the $26 \mathrm{~L}$. barbarum L. from China (BC01-BC26), 3 L. barbarum L. (BJ27-BJ29) from Japan, 12 L. chinense Miller from China (CC30-CC41), and 51 L. chinense Miller samples (CK42CK92) from Korea, pattern recognition analysis was conducted using the peak areas of betaine and one unknown peaks. In the pattern recognition analysis we indicated that all of the samples were largely clustered into two groups, A (L. chinense Miller) and B (L. barbarum L.). This result revealed that the pattern analysis of Lycii Fructus samples had a relationship with species ( $L$. barbarum L. and $L$. chinense Miller) regardless of geographical origin (Fig. 4). Interestingly, although the contents of betaine were similar in the two species of $L$. barbarum $\mathrm{L}$. and $L$. chinense Miller, Hclust could distinguish between $L$. barbarum L. and $L$. chinense Miller. Hence, this pattern analysis results could be used for the quality control of Lycii Fructus.

Acknowledgments This research was supported by a Grant (09112KFDA817) from the National Center for Standardization of Herbal Medicine funded by the Korea Food \& Drug Administration, Republic of Korea.

Open Access This article is distributed under the terms of the Creative Commons Attribution License which permits any use, distribution, and reproduction in any medium, provided the original author(s) and the source are credited.

\section{References}

Adams, M., M. Wiedenmann, G. Tittel, and R. Bauer. 2006. HPLCMS trace analysis of atropine in Lycium barbarum berries. Phytochemical Analysis 17: 279-283.
Duan, H., Y. Chen, and G. Chen. 2010. Far infrared-assisted extraction followed by capillary electrophoresis for the determination of bioactive constituents in the leaves of Lycium barbarum Linn. Journal of Chromatography A 1217: 4511-4516.

Huang, H., X.S. Chen, and Q. Liao. 2010. Determination of the contents of betaine in Lycium barbarum L. by HPLC-ELSD. Chinese Journal of Experimental Traditional Medical Formulae 16: 20-27.

Inbaraj, B.S., H. Lu, C.F. Hung, W.B. Wu, C.L. Lin, and B.H. Chen. 2008. Determination of carotenoids and their esters in fruits of Lycium barbarum Linnaeus by HPLC-DAD-APCI-MS. Journal of Pharmaceutical and Biomedical Analysis 47: 812-818.

Korea Food and Drug Administration. 2007. Korean Pharmacopoeia IX, Part II (K. P.), 913. Seoul: Shinil Books.

Lee, S.M., C.K. Park, B.G. Cho, K.S. Cho, B.S. Min, and K.H. Bae. 2011. A convenient HPLC/ELSD method for the quantitative analysis of betaine in Lycium chinense. Natural Product Sciences 17: 104-107.

Li, X.M., Y.L. Ma, and X.J. Liu. 2007. Effect of the Lycium barbarum polysaccharides on age-related oxidative stress in aged mice. Journal of Ethnopharmacology 111: 504-511.

Liu, L., X.N. Wang, Z. Liu, L.N. Wang, J. Wu, W. Wang, and J.X. Feng. 2011. Effect of Lycium barbarum polysaccharides on angiotensin II-induced senescence of human umbilical vein endothelial cells and expressions of P53 and P16. Nan Fang Yi Ке Da Хие Хие Ваo 31: 1212-1215.

Michels, K.B. 2011. Epigenetic epidemiology, 354-355. New York: Springer.

Peng, Y., C. Ma, Y. Li, K.S. Leung, Z.H. Jiang, and Z. Zhao. 2005. Quantification of zeaxanthin dipalmitate and total carotenoids in Lycium fruits (Fructus Lycii). Plant Foods for Human Nutrition 60: 161-164.

Shin, H.D., J.H. Suh, J.H. Kim, H.Y. Lee, H.Y. Eom, U.Y. Kim, D.H. Yang, S.B. Han, and J.R. Youm. 2012. Determination of betaine in Fructus Lycii using hydrophilic interaction liquid chromatography with evaporative light scattering detection. Bulletin Korean Chemical Society 33: 553-558.

Shin, Y.G., K.H. Cho, J.M. Kim, M.K. Park, and J.H. Park. 1999. Determination of betaine in Lycium chinense fruits by liquid chromatography-electrospray ionization mass spectrometry. Journal of Chromatography A 857: 331-335.

Tang, W.M., E. Chan, C.Y. Kwok, Y.K. Lee, J.H. Wu, C.W. Wan, R.Y. Chan, P.H. Yu, and S.W. Chan. 2011. A review of the anticancer and immunomodulatory effects of Lycium barbarum fruit. Inflammopharmacology 1: 120-125.

The Pharmacopoeia Commission of the People's Republic of China. 2010. Pharmacopoeia of People's Republic of China (C. P.), 232-233. Beijing: The Chemical Industry.

Xu, M.W., X.Z. Yuan, Y.W. Liu, and X.J. Shen. 2006. Determination of betaine in Fufang Guilu granule by HPLC. Chinese Journal of Chinese Materia Medica 31: 159-163.

Zhang, R., K.A. Kang, M.J. Piao, K.C. Kim, A.D. Kim, S. Chae, J.S. Park, U.J. Youn, and J.W. Hyun. 2010. Cytoprotective effect of the fruits of Lycium chinense Miller against oxidative stressinduced hepatotoxicity. Journal of Ethnopharmacology 130: 299-306. 